Original Research Paper

\title{
Performance Evaluation of Handover in WiMax with TCP and UDP as Underlying Protocol
}

\author{
Karam Dhafer Mayoof, Rosilah Hassan, Amjed Sid Ahmed and Ahmed Marwan \\ Research Centre for Software Technology and Management, \\ Network and Communication Technology Lab, Faculty of Information Science and Technology, \\ Universiti Kebangsaan Malaysia, 43600 UKM, Bangi, Selangor, Malaysia
}

Article history

Received: 16-03-2015

Revised: 03-12-2015

Accepted: 09-12-2015

Corresponding Author:

Rosilah Hassan

Research Centre for Software

Technology and Management,

Network and Communication

Technology Lab, Faculty of

Information Science and

Technology, Universiti

Kebangsaan Malaysia, 43600

UKM, Bangi, Selangor,

Malaysia

E-mail: rosilah@ukm.edu.my

\begin{abstract}
The rejection of an ongoing connection/session is serious problem and it degrades the QoS and efficiency of the network rather than rejecting a request for new connection. The impact on performance of handovers is a serious problem in cellular systems that must be addressed. During handover there may be delay in packets and connections may drop. Real time applications i.e., VoIP and streaming video can be adversely affected by such kind of delays. In this study we used TCP and UDP as an underlying protocol for exchange of data between two wireless mobile nodes in a WiMax access network and had evaluated the effect on performance in terms of end-to-end delay and throughput. The results showed that when handover is triggered the transfer window resets to zero causing higher throughput and end-to-end delay for TCP than UDP based on packet size and traffic load.
\end{abstract}

Keywords: WiMax, Mobility, Handovers, TCP, UDP, Performance, Handover Latencies

\section{Introduction}

WiMax is a technology standard for long range wireless communication and Transmission control Protocol (TCP) is a protocol used to establish a connection between two clients and exchange data. In WiMax networks mobility is achieved by continuously switching a call from one WiMax base station to another WiMax base station when the nodes moves from former base station coverage area to the later base station coverage area. The Mobile Subscriber (MS) when wish to join a network the network entry procedure must be followed. The steps that are involved are; scanning for a frequency on a Base Station (BS), the MS performs repeated scanning in order to maintain the connectivity while the MS moves from coverage area of one BS to the coverage area of another BS, the changing of BS's is called a handover. The most significant feature in WiMax networks is the support for mobility, which is one of the fundamental requirements for mobile communication networks. The use of mobiles is the growing trend in communication today, due to the fact that the wireless networks provide sufficient data rates capable to compete with wired connections. Any time anywhere access to web services are in great demand that gives the motivation to have instant messages, emails and multimedia services that are available on mobile phones and PDA's etc. The users also demand for easy and reliable data services with improve QoS (Saeed et al., 2014); on the other hand the service providers try to use the resources optimally maintaining a balance between cost and efficiency. Due to the deployment of applications such as steaming video, music downloads and VoIP there is a demand for high band width. The IEEE $802.16 \mathrm{e} / \mathrm{WiMax}$ standards promise to offer the infrastructure for high speed mobile internet. Also the networks supporting mobility sets requirements for the mobile devices; the fore most is the ability of a device to switch from one BS to another when the user changes its position; maintaining the connection without any disturbances in communication assuring confidentiality between MS, current and new BS's. The handovers needs to be supported even if the user is moving at high speed e.g., travelling on highway or in train. Another issue is the limited power resource of a mobile device since the battery can carry a fix amount of charge and is recharged on regular basis.

Mobility was a major problem with traditional WiMax since it only supported nomadic access. In this scenario the mobile device was able to change the subscriber location without supporting handovers. The IEEE 802.16e has genuinely solves the problem and had provided the capability to WiMax to go mobile. Since adjacent cells use disjoint subset of frequency bands 
consequently negotiation must take place between mobile terminal, the current base station and next potential base station. It is also defined as the switching of frequencies from one wireless cell to another without disconnecting the session. Handover initiation, handover decision and handover execution can be the three phases of handover in which the whole process can be divided for handover mechanisms.

The first two phases (handover initiation and decision) can be comprised of any of the four basic schemes which are: Mobile controlled handover (MCHO), network controlled handover (NCHO), mobile assisted handover (MAHO) and network assisted handover (NAHO). There can be other hybrid schemes evolved from these basic schemes such as mobile assisted network controlled and network assisted mobile controlled handovers. The handover implementation phase pursues the initiation and decision phases. In implementation phase the mobile node establishes handover connections with target network and releases all connections with serving network that involves signaling exchange procedure between mobile node and the network entity (target or serving network). The signaling exchange procedure between the mobile node and network for handover execution can be of two types such as backward and forward. The backward scheme utilizes serving network link for signaling exchange, whereas the forward scheme establishes and uses new signaling link with target network (Esa and Pentikousis, 2009; Li et al., 2010; Lan and Yu, 1995).

The aim of this paper is to evaluate the Performance of Handover in WiMax with TCP and UDP protocols. Performance matrices in term end-to-end delay and throughput, which is defined as the average rate of successful message delivery over a communication channel (Zahian and Hassan, 2011a; 2011b), were used in this study against packet size and traffic load. This paper contains six main sections. Handover types is the first section we talked about followed by the related works, after which we described the model we used for the purpose of simulation in model description section directly followed by the simulation scenario section. Before finally we conclude the paper in conclusion part we present the results in the results section.

\section{Handover Types}

Handover is required when a MS moves from one BS area to another BS area or when the signal quality degrades to a level where it is not possible to maintain the connection. Sometimes handovers occur within the same BS area between channels. This handover is called inter cell handover while the former one is called inter cell handover. Handovers are also possible between different technologies; known as vertical handovers while the former are known as horizontal handovers. The later were defined to be handovers within a single technology while the former one is handovers between different/multiple technologies. There can be varied reasons for the handovers to occur, below are few of the reasons:

- At the edge of the cell the signal strength is not enough to maintain the communication

- The capacity of a BS is full and there is no room more traffic

- There is a co channel interference from neighboring cells

- In vertical handovers there a handover occurs when there is a faster and cheap technology available

- MS behavior changes

The 802.16e supports three types of handovers methods; Hard Handover (HHO), Micro Diversity Handover (MDHO) and Fast Base Station Switching (FBSS) (Pardi et al., 2011).

\section{Hard Handover}

The hard handover is break before make i.e., the connection with the current BS is broken before making a connection with the new BS. The advantage of $\mathrm{HH}$ is that the access signaling is avoided during the hand over but it may take longer time for the connection to get back to normal. The mobile node listens to link layer messages while connected to $\mathrm{BS}$, in case a neighbor BS advertisement is received.

\section{Macro Diversity Handover}

In mobile WiMax MDHO is an optional handover scheme and is supported by both the BS and the MS. A list of BS's having the capability of MDHO is maintained by MS; the group is called Diversity Set. An anchor BS is defined in the diversity set in BS. There can be cases when the MS can reach the BS's but the signal strength is too low for real traffic. An MS while approaching towards a BS a point will reach that the signal strength becomes strong enough and the BS will eventually be added in the diversity set.

\section{Fast Base Station Switching}

The principles for FBSS are same as MDHO; both the MS and BS should be capable of supporting the FBSS. MS and BS both keeps the diversity set maintained but the MS only communicates with one BS in the diversity set. The active/current BS is named as anchor BS. The focus is only on one BS in the FBSS for communication; this includes signaling traffic as well. The data is received by all the BS's in the diversity set addressed for MS but only one of them transmits data over the air interface while others ultimately drop the received packets.

\section{Related Work}

With the ever increasing growth of wireless technologies, the objective to provide proficient 
connectivity is still a challenging job. As almost every wireless network poses several problems, that includes limited bandwidth, coping with bandwidth fluctuations and corrupted or lost data, considerable amount of work has been carried out and a number of solutions has been proposed. The most common transmission protocol used over the internet is the TCP. Due to the packet loses regular TCP is not suitable for wireless networks; these loses are more due to unreliable links than due to congestion. Hwang et al. (2005) TCP performance is carried out and a cross layer design is proposed to improve performance. To adaptively control retransmission decision between sender and receiver, control packet size and packet amount feedback information about the channel state is used. The authors have tried to reduce the retransmissions to improve performance. Zhang et al. (2012) TCP overall performance is performed and the cross layer design is actually proposed to improve performance. To adaptively command retransmission conclusion between sender along with receiver, control package size along with packet volume a feedback details about the funnel state is employed. The authors have tried to lessen the retransmissions to improve performance. Khademi et al. (2012), authors studied the performance of TCP coupled with different commonly deployed RA mechanisms. Authors found that most conventional RA mechanisms are unable to separate frame errors due to collisions from channel noise/interference and will respond to them negatively to some extent; they also found that various events related to downlink TCP, uplink TCP can be adversely affected by collisiontriggered rate downshifts that some RA schemes exhibit even under perfect channel conditions or in low-noise environment in which the resulted negative uplink behavior could lead to almost equal performance as in the downlink case.

By projecting data latency pattern a new methodology is introduced in (Pardi et al., 2011) to improve performance of handover's in WiMax networks. In (Shidhani and Leung, 2010) UDP is used as a transmission protocol with different modulation schemes; the author concluded that with increased number of nodes the throughput increases but the delay increases; also 64QAM turns out to be efficient modulation scheme than QPSK and BPSK.

The interworking of UMTS and WiMAX networks has provisioned the cost effective and global roaming broadband wireless Internet access. The design of efficient Inter and Intra WiMax handovers in the interworking paradigm is a challenging job. These Handovers must be secure and instantaneous at the same time. An attempt is made in (Naqvi et al., 2012) to address the aforementioned issue by designing Intra and Inter WiMAX handover protocols capable of operating in the UMTS-WiMAX interworking architecture and performs mutual pre-authentication between the mobile station and the target network prior to handover. The author's argue that due to the pre-authentication procedure adopted the efficiency has improved considerably as there are less handover signaling messages.

A lot of efforts have been put to improve the performance of mobile WiMax by suggesting different techniques to reduce signaling messages while a handover is in process. In this study an ns-2 simulation is carried out to see the performance of handover in a mobile WiMax network with underlying TCP and UDP protocol.

\section{Model Description}

We have considered a scenario where there are mobile nodes uniformly distributed over the plane connected with two base stations i.e., BS0 and BS1. As for the mobile users the handover should be fast enough in a way that the ongoing video or voice session is not interrupted or to an extent that the user does not notices it. In the present scenario when a mobile station moves away from area of one base station towards another base station area or the signal strength has decreased to an extent where it is not possible to continue the communication; a handover is initiated in a way that there is minimum loss to data and the existing connections are not disturb.

We have considered two BS's as for a handover to take place at least two BS's are required, current BS and new BS with which the connection has to be made. There are two types of handovers that are considered one is inter cell with the BS say BS1 where the BS1 address is 1.1 handover where the handover occurs between channels i.e., channels are switched and other is intra handover where the BS's are changed. The mobile node is basically registered.0. During the simulation the mobile node moves towards the common coverage area of BS0 and BS1, BS1 has the address 2.1.1. When the mobile node sees (detects signals from BS1) the coverage area of BS1 and the signal strength of BS0 becomes weaker the mobile node handovers to the BS1 and acquires the new care of address from BS1.

The new address (care-of-address) of the mobile node becomes 2.1.2 and all the connections associated with mobile node are updated about the new (care-of) address. The overlapped coverage area is the place where handover occurs.

Handover between two technologies are also possible known as Media Independent handover which is out of the scope of this paper. The model diagram is shown in Fig. 1.

The simulation of the proposed model is carried out using ns 2 and the parameters that have been observed are Throughput, End to End delays, Jitters, Drops and Handover Latencies. 


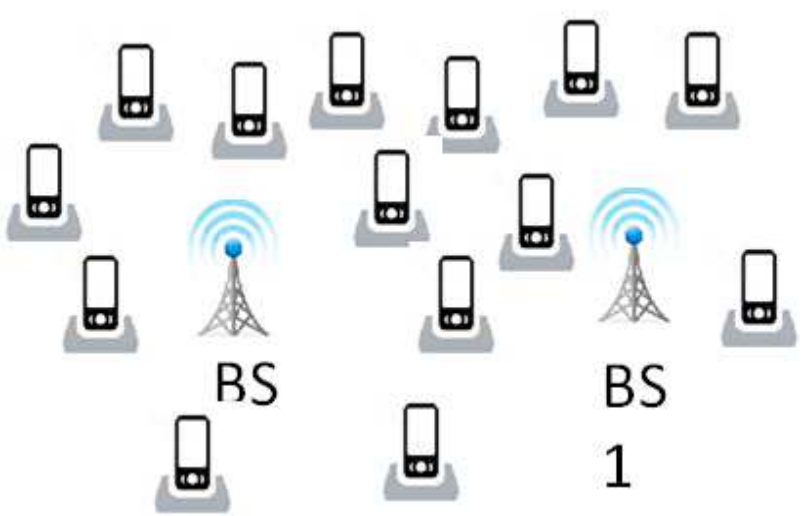

Fig. 1. The model of handover between two technologies

\section{Simulation Scenario}

The execution process of handover is categorized in two phases i.e., preparation and action as shown in Fig. 2. In the first phase the negotiation of expected quality of service (QoS) takes place via backbone messages for pre handover notification. When the signal strength decreases to a point where it is not possible to continue the communication a hand over request is initiated by the Mobile Subscriber (MS) by sending a handover request to the Base Station (BS), the BS than select from a list of available BS's the best that can offer required resources and QoS. In Fig. 3 a conceptual boundary that differentiates between BSs are given.

As illustrated in Fig. 4, the target BS requests for the MS information with MS Info REQ message, the current BS then forwards the info with MS Info RSP message. The Authentication and Service Authorization Server (ASA) then send the authorization messages with MS Authorization RSP message to the target BS. The hand over process optimization starts at the MS; where it decides which steps of network entry would be required during handover process. The action phase of the handover continues with the message of MS to the serving BS of its decision of for Handover and the link with the serving BS is disconnected and it no longer communicate with serving BS.

The Hand Over (HO) is triggered and an initiation process for network entry with the target BS is started. The break time of $\mathrm{HO}$ starts when the sending $\mathrm{HO}$ indication message is sent and ends when the MS registration with the target $\mathrm{BS}$ is completed. This period is known is HO latency period. The MS obtains the Downlink (DL) and Uplink (UL) transmission parameters and synchronize with downlink transmissions of target BS. The MS and target BS conduct a $\mathrm{HO}$ ranging procedure, pursued by authorization, key exchange and negotiation of basic capabilities and lastly by registration with the target BS.

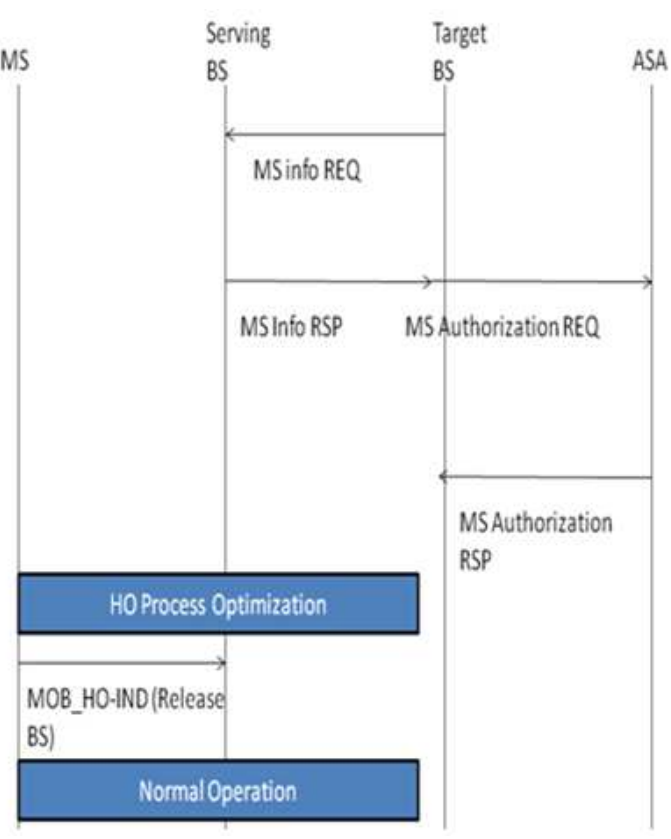

Fig. 2. Handover process

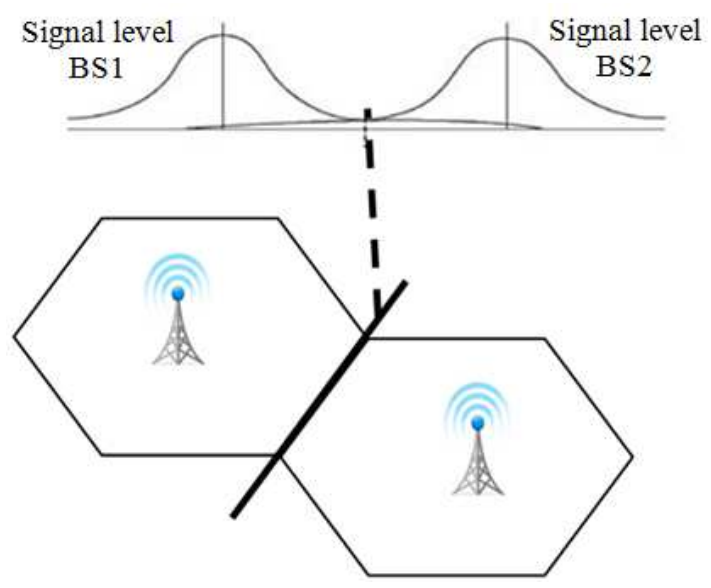

Fig. 3. Handover process

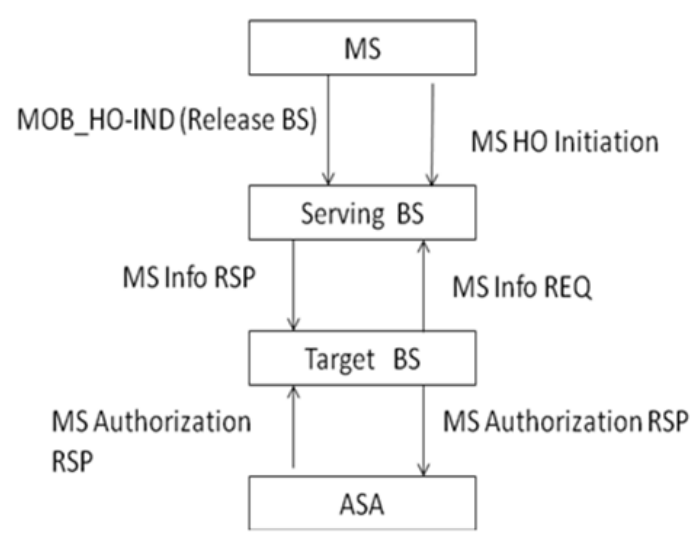

Fig. 4. Simulation scenario 


\section{Results}

The simulation of the network is carried out using network simulator 2. One of the advantages of TCP is congestion control, in scenarios where congestion is a problem, TCP was be given preference but TCP being a reliable service and delays will be encountered whenever packet loss or bit error occurs. The delay is the cause of a retransmission of packet, plus any successive packets that are sent already. These phenomena can be a major source of throughput. All the algorithms used by TCP for congestion control uses packet loss as an indication of congestion. In such situation, all alter packets TCP will send before waiting for acknowledgment of alter packets. The alteration affects bandwidth and changes delay and can also be source of throughput in TCP networks.

The variation in packet arrivals for UDP is less than and TCP varies as the end to end delay is caused by retransmissions of packets when a packet is size increases which results in UDP delays compared to TCP as shown in Fig. 5. The end-to-end delay does adversely affect the performance of network. When TCP is used as transmission protocol; data rates are higher, the reliability of data transfer is higher, the losses are at its minimum but the drawback is that there are higher delays because whenever a handover is triggered the window size is reset to zero. On the other hand, UDP is connectionless protocol and is good for voice and video streaming and real time applications but there are losses; that requires retransmissions and can overload the network.

As the UDP is connectionless protocol, the throughput is lower as compared to TCP shown in Fig. 6. The sudden increases in packet size vary in UDP while it appears to be stable in TCP with higher throughput compared to the UDP.

In Fig. 7, the end-to-end delay assessed by the traffic load appears to be moderately stable in TCP more than UDP as shown in fig below.

In addition, the throughput of UDP appears to be better than TCP with substantial increases of the traffic as shown in Fig. 8. The number of packets for TCP load is higher than UDP as the transmission rate is slower as compared to TCP, also the window size decreases when handover is triggered hence reducing rate.

With the increase in data rate the traffic load increases, so are the retransmissions. The traffic load is higher in TCP as compared to UDP.

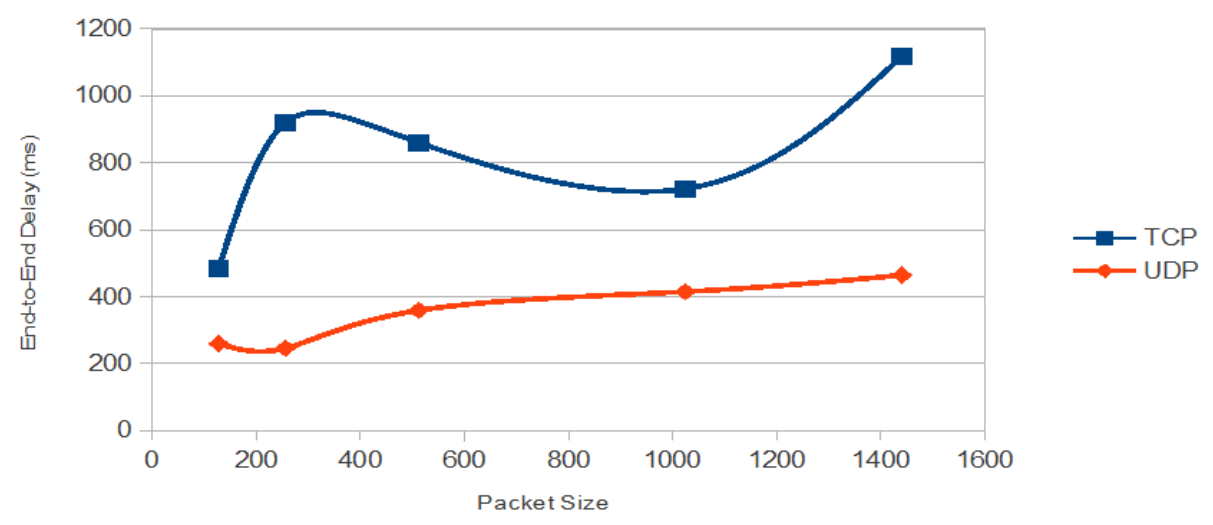

Fig. 5. TCP and UDP end to end delay based on packet size

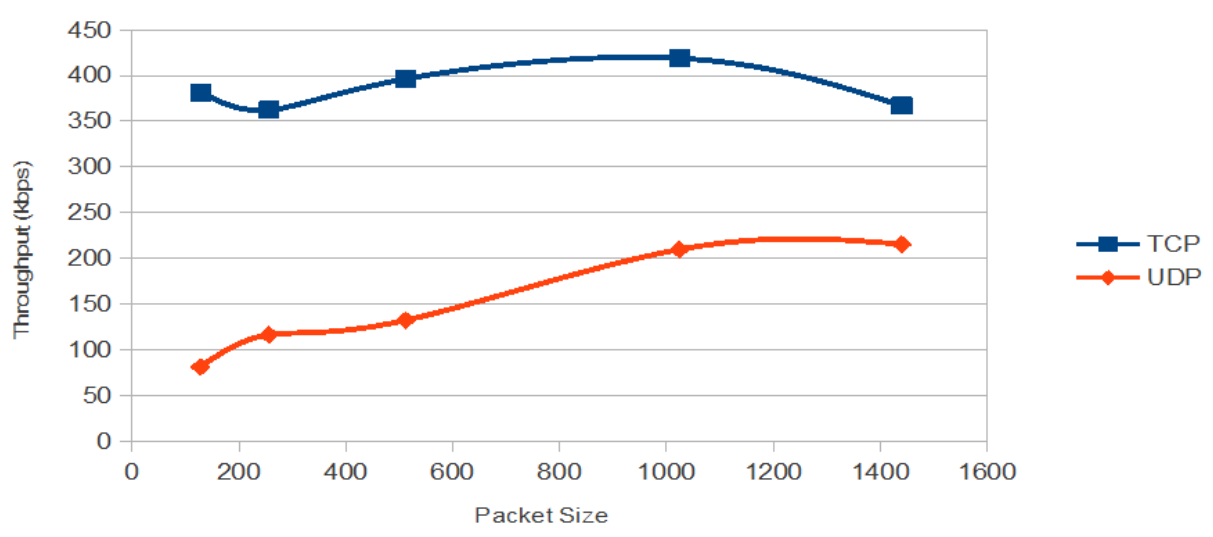

Fig. 6. TCP and UDP end to end delay based on packet size 


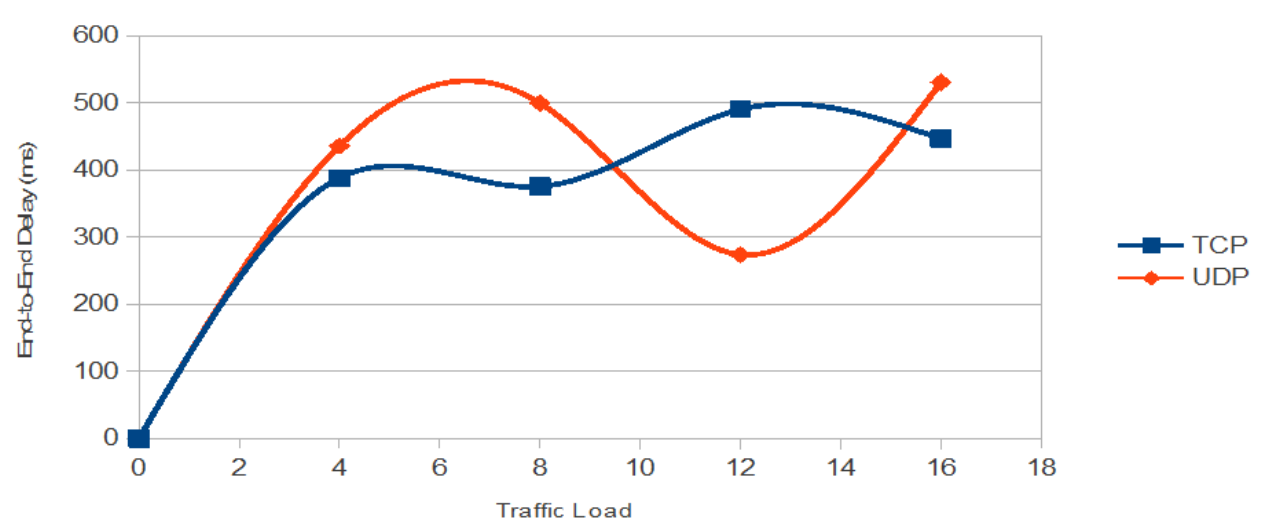

Fig. 7. TCP and UDP throughput based on traffic load

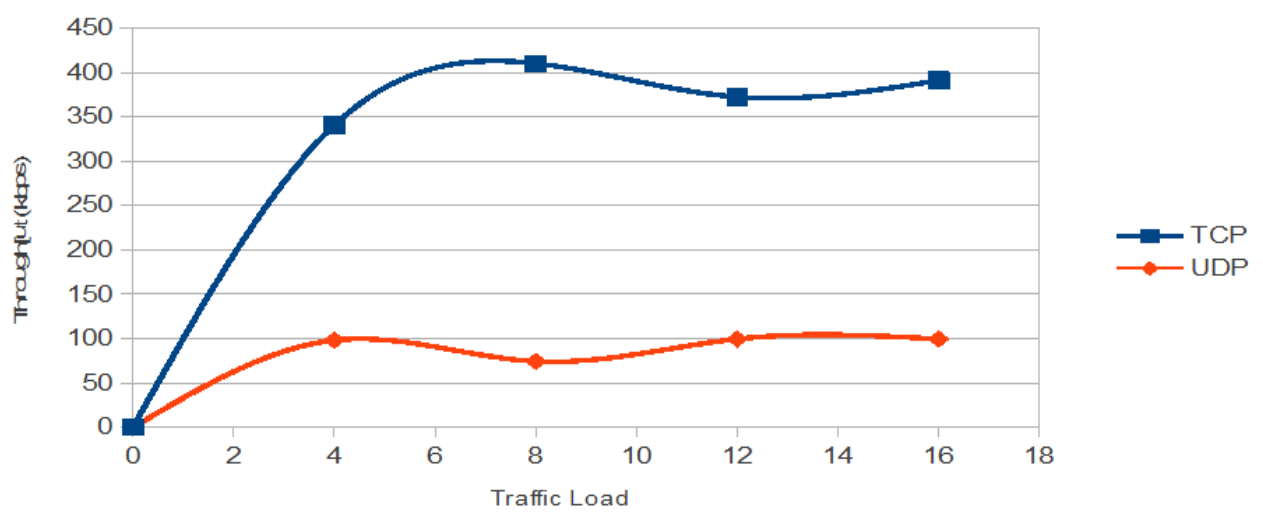

Fig. 8. TCP and UDP throughput based on traffic load

\section{Conclusion}

In this study we evaluate performance of handover in WiMax networks. We used TCP and UDP as an underlying protocol for exchange of data between two wireless mobile nodes in a WiMax access network. We had evaluated the effect on performance in terms of endto-end delay and throughput. A simulation for WiMax network was carried out using Network Simulator 2, from the simulation results we can conclude that UDP gives better results when it used for real time data in WiMax networks than TCP; there will be loss of packets/data when UDP is used but on the other hand throughput will be higher than the TCP as whenever there is a handover the TCP window size is reset which in turns decreases the throughput, also the hand over latencies for TCP are higher than UDP; delay will be greater than UDP as in TCP the window size is reset whenever a handover occurs which in turns reduces the amount of data to be transmitted therefore the packets will take longer as obvious from the aforementioned graphs. A future research could be conducted to examine the connectivity of WiMAX to other wireless networks by considering shorter handover interruption time in which it believe to effect the performance of packet handling while transformation.

\section{Acknowledgment}

The research is conducted at Network and Communication Technology Laboratory (www.nctlab.net) at Faculty of Information Science and Technology (FTSM), Universiti Kebangsaan Malaysia (UKM). The lab is led by the second author, the first, third and fourth authors are researchers at the lab.

\section{Author's Contributions}

The research is primary led by the first author, giving the area of research and highlights the problem statement and research question for the researchers inside Network and Communication Technology Laboratory (NCT). The second author preparing the layout of the paper and structuring how the contents should be. In addition to preparing the simulation environment and scenarios and implementing the experiment. The third authors was responsible for collecting materials and writing the literature review in 
addition to editing and proof reading of the paper. Lastly the fourth author was responsible for the analysis and conducting a part of literature review as well.

\section{Ethics}

This paper is original and contains genuine materials that never published before. The corresponding author confirms that all of the other authors have read and approved the manuscript and no ethical issues involved. All authors as well have no objection for the article to be open access for public.

\section{References}

Esa, P. and K. Pentikousis, 2009. IEEE 802.21: Media independent handover services.

Hwang, J., S.W. Son and B.H. Rhee, 2005. Improving TCP performance over WiMAX networks using cross-layer design. Proceedings of the 3rd International Conference on Convergence and Hybrid Information Technology, Nov. 11-13, IEEE Xplore Press, Busan, pp: 83-88. DOI: 10.1109/ICCIT.2008.316

Khademi, N., M. Welzl and S. Gjessing, 2012. Experimental evaluation of TCP performance in multirate 802.11 WLANs. Proceedings of the IEEE International Symposium on a World of Wireless, Mobile and Multimedia Networks, Jun. 25-28, IEEE Xplore Press, San Francisco, pp: 1-9.

DOI: 10.1109/WoWMoM.2012.6263696

Lan, Y.R. and T. Yu, 1995. A dynamic central scheduler load balancing mechanism. Proceedings of the IEEE 4th Annual International Phoenix Conference on Computers and Communications, Mar. 28-31, IEEE Xplore Press, Scottsdale, AZ, pp: 734-740. DOI: 10.1109/PCCC.1995.472412

Li, B., W.X. Shi and N. Li, 2010. A hierarchical semicentralized architecture for load balancing of heterogeneous wireless networks. Proceedings of the 2nd International Conference on Networks Security Wireless Communications and Trusted Computing, Apri. 24-25, IEEE Xplore Press, Wuhan, Hubei, pp: 28-31. DOI: 10.1109/NSWCTC.2010.144
Naqvi, A.N., A.M. Abbas, T.A. Chouhan and Z. Hussain, 2012. Performance evaluation of fixed and mobile WiMAX networks for UDP traffic. Int. J. Advanced Res. Comput. Commun. Eng.

Pardi, M.S., S. Alam, M.D. Baba and M. Ibrahim, 2011. Analysis of handover performance in mobile WiMAX networks. Proceedings of the IEEE Control and System Graduate Research Colloquium, Jun. 27-28, IEEE Xplore Press, Shah Alam, pp: 143-149. DOI: 10.1109/ICSGRC.2011.5991847

Saeed, S., M. Ismail, R. Hassan and A. Al-Hemyari, 2014. Flow-based admission control and QoS maintenance for real time traffic over MANETs. Int. Rev. Comput. Software, 9: 1712-1720. DOI: 10.15866/irecos.v9i10.3929

Shidhani, A.A. and V.C.M. Leung, 2010. Enhancing the performance of secured handover protocols in UMTSWiMAX interworking. Springer J. Wireless Netw., 16: 1929-1943. DOI: 10.1007/s11276-010-0236-4

Zahian, I. and R. Hassan, 2011a. A performance study of various mobility speed on AODV routing protocol in homogeneous and heterogeneous MANET. Proceedings of the 17th Asia-Pacific Conference on Communications (APCC), Oct. 2-5, Sabah, pp: 637-642. DOI: $10.1109 /$ APCC.2011.6152886

Zahian, I. and R. Hassan, 2011b. Effects of packet size on AODV routing protocol implementation in homogeneous and heterogeneous MANET. Proceedings of the 3rd International Conference on Computational Intelligence, Modelling and Simulation (CIMSiM), Sept. 20-22, IEEE Xplore Press, Langkawi, pp: 351-356. DOI: 10.1109/CIMSim.2011.70

Zhang, L., T. Okamawari and T. Fujii, 2012. Performance evaluation of TCP and UDP during LTE handover. Proceedings of the Wireless Communications and Networking Conference (WCNC), Apri. 1-4, IEEE Xplore Press, Shanghai, pp: 1993-1997. DOI: 10.1109/WCNC.2012.6214116 